\title{
QoS routing in cluster OLSR by using the artificial intelligence model MSSP in the big data environnment
}

\author{
Jawad Oubaha ${ }^{1}$, Noureddine Lakki ${ }^{2}$, Ali Ouacha ${ }^{3}$ \\ ${ }^{1,2}$ Department of Computer Engineering, University of Mohammed V, Rabat, Morocco \\ ${ }^{1,3}$ IPSS Team Computer Science Department, University of Mohammed V, Rabat, Morocco
}

\begin{tabular}{|c|c|}
\hline Article Info & ABSTRACT \\
\hline Article history: & \multirow{9}{*}{$\begin{array}{l}\text { The most complex problems, in data science and more specifically in artificial } \\
\text { intelligence, can be modeled as cases of the maximum stable set problem } \\
\text { (MSSP). this article describes a new approach to solve the MSSP problem by } \\
\text { proposing the continuous hopfield network (CHN) to build optimized link } \\
\text { state protocol routing (OLSR) protocol cluster. our approach consists in } \\
\text { proposing in two stages: the first acts at the level of the choice of the OLSR } \\
\text { master cluster in order to quickly make a local minimum using the CHN, by } \\
\text { modeling the MSSP problem. As for the second step, the objective is the } \\
\text { improvement of the precision making a solution of efficient at the first rank of } \\
\text { neighborhood as a linear constraint, and at the end, to find the resolution of } \\
\text { the model using the CHN. We will show that this model determines a good } \\
\text { solution of the MSSP problem. To test the theoretical results, we propose a } \\
\text { comparison with a classic OLSR. }\end{array}$} \\
\hline Received Oct 24, 2019 & \\
\hline Revised Mar 22, 2021 & \\
\hline Accepted Apr 19, 2021 & \\
\hline Keywords: & \\
\hline Big Data & \\
\hline Routing & \\
\hline Quality of Service & \\
\hline MSSP & \\
\hline MANET & \\
\hline
\end{tabular}

MANET

OLSR

This is an open access article under the CC BY-SA license.

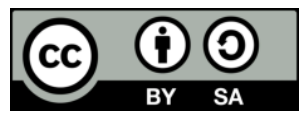

Corresponding Author:

Jawad Oubaha

Department of Computer Engineering

University of Mohammed V, Rabat

Madinat Al Irfane Agdal, Rabat, Morocco

Email: j.oubaha@um5s.net.ma

\section{INTRODUCTION}

The communication in wireless networks in mobile ad hoc network (MANETs) [1] remains a great challenge because of its specificity, taking into account the random mobility of resources and their limitation, the range of each node and also the nature traffic exchanged. Hence the routing protocol comprises the multicriteria grouping the aforementioned parameters including the specificity of abrupt changes and constraints of MANETs. A reinforcement learning becomes a very important element to provide a network strategy is to render the information about the metrics to optimize the operation of the routing protocol. subsequently, this information collected allowing the calculation of the k-hop of the routes in order to select the best one, which meets the performance requirements of the quality of service. Unfortunately the disadvantage of the usual methods are static applicable on all nodes without any change. Of this effect, this work proposes the formulation of the maximum stable set problem MSSP, a metric changes according to the applied position. Indeed, the introduction into the routing process, the dynamic notion of multi-objective in the construction process of all multipoint relays (MPRs) [2], [3].

This article is organized as follows: two sections: the first one begins with brief summary of our work and an introduction containing general concepts on networks MANETs at the end of this introduction we present the problematic of our article. Then, we will present the principle of MSSP formulation and different messages exchanged in optimized link state protocol routing (OLSR). The second section begins with the contribution of this paper in which we explain our approach based on several metrics to improve 
QoS in the network MANETs. Then simulation results with its interpretations, finally finishing with a conclusion of this article.

\section{OLSR OVERVIEW}

\subsection{Principles of OLSR}

OLSR is a proactive wireless routing protocol, a hierarchy between nodes based on neighborhoodtype link state. In fact, OLSR optimizes operation by distributing and reducing the number of control messages. For this, it chooses the multipoint relay (MPR) among the neighborhood constituting the cluster, these MPRs manage the control messages [4].

\subsection{Messages exchanged}

OLSR is based on the periodic exchange of control messages (Hello, TC, MID, HNA) to preview the status of the links [5], step by step the management of the entire network, the sending of messages at regular time intervals. This Figure 1 illustrates the message exchange and its type:

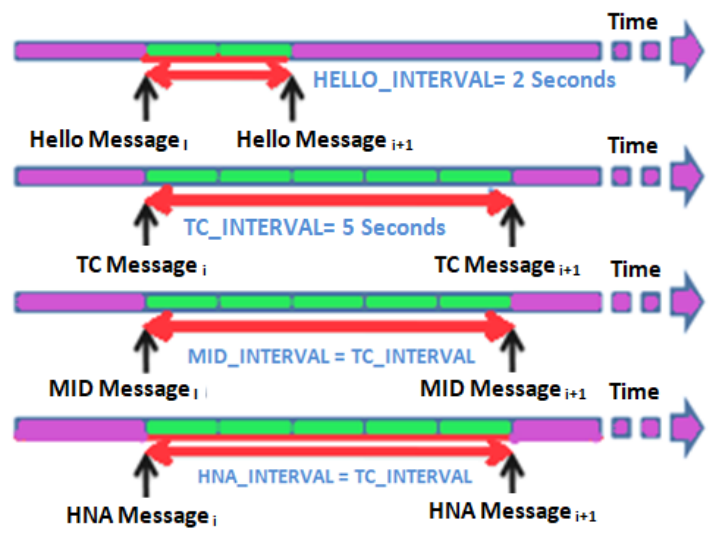

Figure 1. The exchange messages in the OLSR protocol for time intervals

a. Multiple interface declaration MID message

Each node periodically sends information messages describing its neighborhood [6] by distributing the entire network in MID messages as shown in Figure 2.

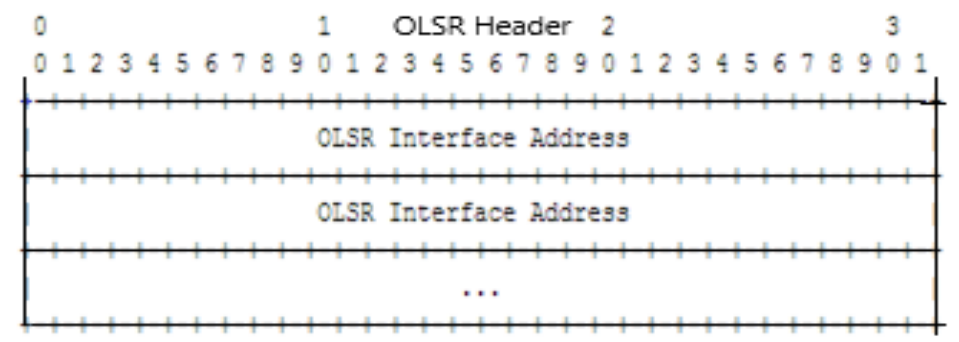

Figure 2. Multiple interface declaration MID message structure

MID_MESSAGE contains:

- TTL (Time To Live): on 255 to prevent loops,

- MID_HOLD_TIME: (OLSR Interface Address) contains the address of an OLSR interface of the node.

b. HELLO messages

The periodic exchange of "Hello" message aims to discover neighboring nodes and then chooses the MPR as shown in Figure 3. HELLO messages include [7]:

- $\quad$ The time interval between two HELLO messages from the originating node, 
- $\quad$ Perform routing operations for the address list following this field.

- $\quad$ Specify the nature of the relationship between the original message and the node address list.

The link code specifies both the link type (unspecified, asymmetric, symmetric, lost) and the type of neighbor at the other end of the link (symmetric, MPR, non-neighbor).

c. Topology control (TC) message

MPR is a responsible for distributing the message topology control (TC) messages throughout the network as shown in Figure 4. The sequence number (ANSN) of the TC messages incremented after each change of node topology, this message also includes the address list [8].

d. Host and network association (HNA) message

The HNA message, is an additional OLSR protocol message [9], ensures routing and communication between an OLSR network and another network as shown in Figure 5.

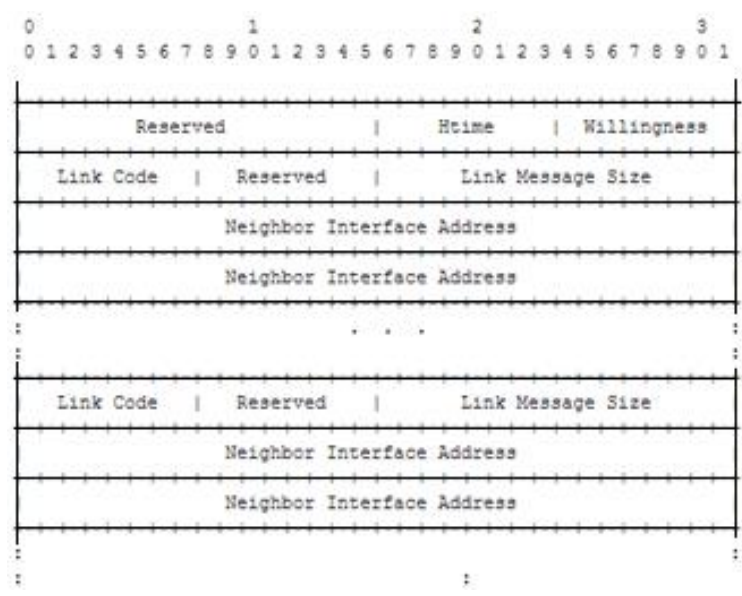

Figure 3. HELLO message

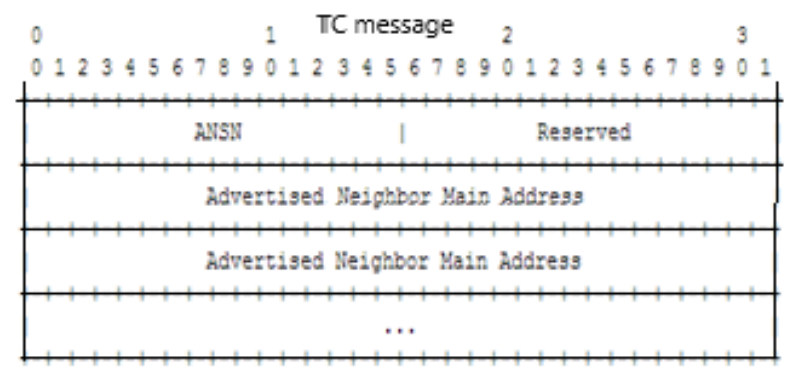

Figure 4. TC message

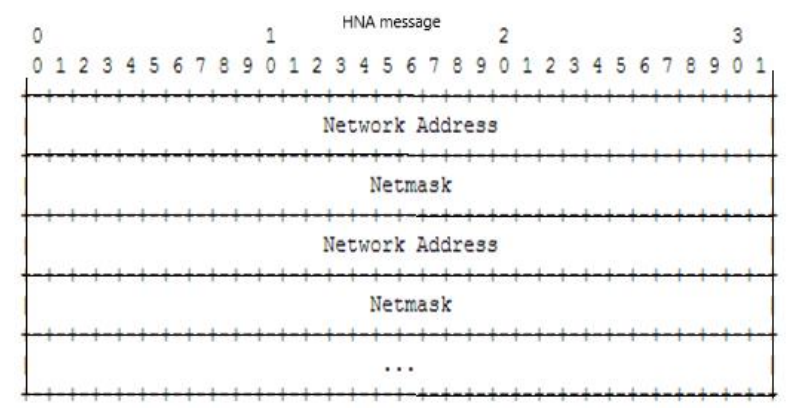

Figure 5. Host and network association message structure 


\subsection{Multipoint relay (MPR) election}

After MPR selection, its own set of MPRs has a symmetrical link with its neighbors. Any MPR is calculated so that from this set it is possible to reach all two-hop neighbors. Figure 6 shows the algorithm for calculating MPRs [10], [11] and described in more detail in RFC 3626.

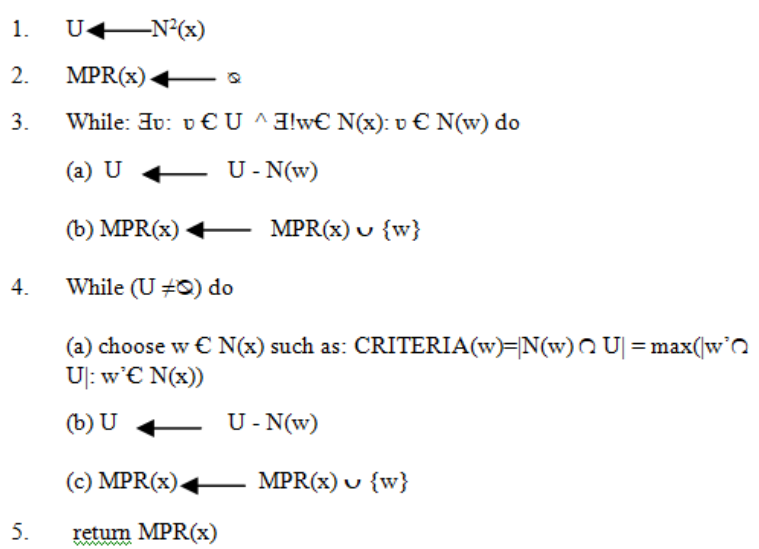

Figure 6. Selecting standard MPRs algorithm

\section{APPROACHES IN BIG DATA ANALYTICS FOR QUALITÉ OF SERVICE}

Big data analysis is one of the most advanced technologies which understands huge volume of data with great variety. The use of information on social media or network equipment represents a real challenge with the evolution of technology to anticipate the good management of resources such as the recommendation system, customer preferences and clustering, to improve sales. This data store that stores a huge volume of data in the form of historical data such as previous system details [12], [13].

The creation of ultra-dimensional data models ensures the differentiation of flows with better affinity, this operation provides real-time decision anticipation and predicting the need. The Hadoop ecosystem of big data design stores large sets of heterogeneous data and analyzes them at a high speed, as part of this work Hadoop will analyze the information gathered from the network and we will define an efficient algorithm in the form of a set of rules and regulations in the flow. It controls the data routing and the nodes positions by comparing the models of the data sets in the data bases which can be processed by the big data analytical.

MapReduce is a frame work of Hadoop and it has big data sets which are stored and processed by big data analytical engine by using the set of algorithms in the form of clustering and it can be computed by using the parallel processing technique [14]. The Hadoop ecosystem is composed of a Map procedure (method) which provides filtering and sorting (such as sorting products by price) and a Reduce method which aggregates in the form of a summary operation (such as counting the number of products that have the same price), and takes the output of "Map" as input and combines these data sets into a smaller set of sets [15], [16]. The "MapReduce system" monitors the distributed processing on multiple servers, with parallel operations, orchestrating all data exchanges between different parts of the system, and fault tolerance with redundant chunks. Map reduce is a processing platform and program model for computing distributed programmed in java [17].

Based on the survey of the papers, the MapReduce algorithm uses on the data sets in the input of the algorithms such as neural algorithm, K-Mean algorithms, clustering algorithms and classification in the data sets. Many research papers are improved the QoS in the network by using the big data analytics scheme which has the efficient and effective process of holding the data sets.

\section{FORMULATION OF THE MAXIMUM STABLE SET PROBLEM}

At the end of the last century, Hopfield worked on neural networks, wrote articles that were written about, and brought a new aspect to the field. this work showed that computational problems [18] could be treated with physical systems. Indeed, the energy function approach is integrated into the optimization process, linear programming problems, and image processing problems. this new era has made it possible to deal with several pending problems such as graph recognition and pattern recognition [19], [20]. 
$x i \begin{cases}1 & \text { if } v_{i \in S} \\ 0 & \text { Othewise }\end{cases}$

In this paper, our objective is to apply the problem of the maximum stable set (MSSP) by integrating the continuous Hopfield network. at the first, the consistency step is to represent or "map" the MSSP problem in the form of an energy function associated with the continuous Hopfield network, according to this model, this mapping step becomes easy and more general. In the next section, we will present $0-1$ quadratic programming of the modeling formulation of the MSSP problem.

Given an undirected graph $G=(V, E)$ with $V=\{v 1, v 2 \ldots, v n\}$. A set of nodes $\mathrm{S}$, such that the nodes of $\mathrm{S}$ are in non-adjacent pairs, represents a stable set of a graph $\mathrm{G}$. the objective is to find a stable set of maximum size of the maximum stable set formulation (MSSP). The quadratic linear representation facilitates the resolution of this MSSP problem via the CHN [21], [22].

For each node $v_{i}$ of the graph $\mathrm{G}$, with $S C V$ a stable set of nodes, we take the binary variables $x_{i}$ such as:

- $\quad$ The mathematical programming model; the objective function is:

$$
f(x)=-\sum_{i=1}^{n} x i
$$

- $\quad$ Two adjacent nodes $v_{i}$ and $v_{j}$ not be in the set $S$ :

$$
\begin{aligned}
& \left(v_{i}, v_{j}\right) \in E \rightarrow x_{i} x_{j}=0 \\
& h(x)=\sum_{i=1}^{n} \sum_{j=1}^{n} b_{\mathrm{ij} . x i x j} \\
& \text { With: } b_{i j}=\left\{\begin{array}{c}
1 \text { if }\left(v_{i}, v_{j}\right) \in E \\
0 \text { Othewise }
\end{array}\right.
\end{aligned}
$$

Indeed, the MSSP problem can be represented in the following algebraic form:

$$
(Q P) \quad \begin{cases}\text { Min } & f(x)=-\sum_{i=1}^{n} x_{i} \\ \text { Subject to } & \\ & h(x)=\sum_{i=1}^{n} \sum_{j=1}^{n} b_{i j} x_{i} x_{j}=0 \\ & x \in\{0,1\}^{n}\end{cases}
$$

Example 1

Graphs built using the MSSP, are undirected graphs as shown in Figure 7. We have a graph with eight nodes and nine edges, which represents "tree stable sets":

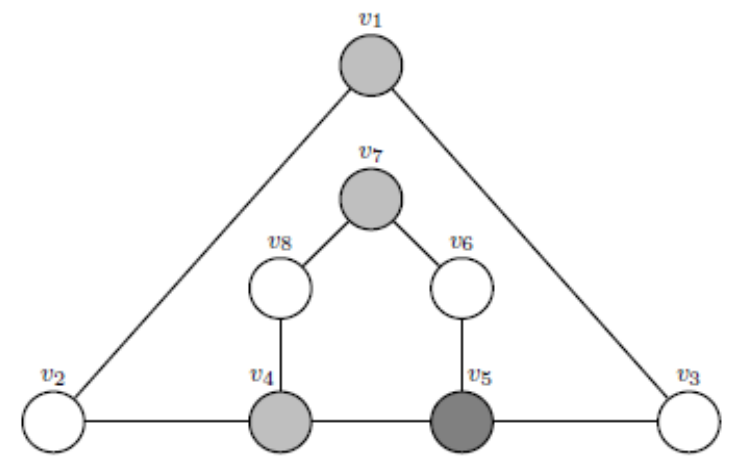

Figure 7. Example of a graph containing tree stable sets

Int J Artif Intell, Vol. 10, No. 2, June 2021: 458 - 466 
The modelization graph with MSSP and a quadratic programming:

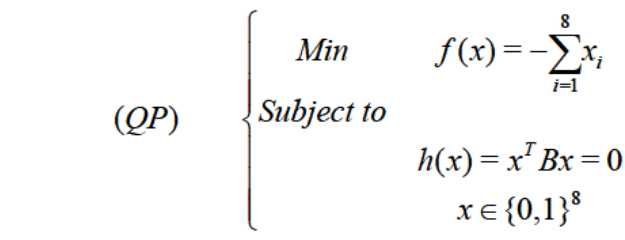

$$
\begin{aligned}
& \left(\begin{array}{llllllll}
0 & 1 & 1 & 0 & 0 & 0 & 0 & 0 \\
1 & 0 & 0 & 0 & 0 & 1 & 0 & 0 \\
1 & 0 & 0 & 0 & 0 & 0 & 1 & 0
\end{array}\right) \\
& \begin{array}{llllllll}
1 & 0 & 0 & 0 & 0 & 0 & 1 & 0
\end{array} \\
& B=\mid \begin{array}{llllllll}
0 & 0 & 0 & 0 & 1 & 0 & 0 & 1 \\
0 & 0 & 0 & 1 & 0 & 1 & 0 & 0
\end{array} \\
& \begin{array}{llllllll}
0 & 1 & 0 & 0 & 1 & 0 & 1 & 0
\end{array} \\
& \begin{array}{llllllll}
0 & 0 & 0 & 0 & 0 & 1 & 0 & 1
\end{array} \\
& \begin{array}{llllllll}
0 & 0 & 0 & 1 & 0 & 0 & 1 & 0
\end{array} \\
& \text { and } \quad x=\left(x_{1}, x_{2}, x_{3}, x_{4}, x_{5}, x_{6}, x_{7}, x_{8}\right)^{T}
\end{aligned}
$$

To solve this model, the interior point, semi-defined relaxations [6] and Lagrangian relaxations [17] are tested and approved methods. In this article, we present the continuous Hopfield network to solve the problem of quadratic constraints. In this section, the minimization of the linear function subjected to quadratic constraints as a 0-1 quadratic programming with the modeled MSSP problem [23].

\section{CONCEPTION AND INTEGRATION OF THE MSSP IN MANET OLSR CLUSTER}

In this paper [24], a broadcast storm could enter the hierarchical topology of the MANET network by hampering the operation and clustering of mobile nodes and also infrastructure-based mobiles. This broadcast storm bored the quality of service of wireless toplology and circulates a reproduced message in the same broadcast domain. Thus, this type of broadcast must be corrected to resume the network to its normal operating state. In this model, we have proposed an intelligent approach, which intercepts any kind of loop, then quickly stops it in a loop step by step the tree topology. The evaluation of the loop resolution method performed in comparison with the hybrid method and also the basic tree topology. After implementation, we found that this method is strong against abrupt change in toplology compared to other methods.

The researcher deals with the communication between a large topology wireless sensors and MANETs networks. Analyzing the effect of mobile networks on the critical transition range, as the handover for asymptotic connectivity, in a multi-level cluster networks in comparison with stationary networks [25]. By integrating the k-hop criterion, all messages sent from a cluster sending station could be transmitted to the cluster master in the k-hops, and thus the transmission delay is limited for the finite $\mathrm{k}$. First, the article defines the critical transition range for the communication of mobile k-hop clusters, these move with a stochastic mobility model. Second, it compares the critical transition range for with a stationary k-hop cluster networks. In addition, fixed and mobile networks are studied in the same way. Thus the transmission power is an inseparable criterion, is studied against the effect of the average energy consumption per flow of different network approaches.

In this article, to use the bandwidth in a rational way, and to save the energy capacity, to keep the minimum security, the disorderly movement of nodes causes problems related to the operation, more particularly in high mobility networks, indeed the nodes frequently change its position. To optimize the constitution of the cluster, improve OLSR routing and ensure good performance, particularly in a big data, in this article, we aim at the density of neighboring nodes with the MSSP formulation to a better manage the MANET cluster. Thus, in our optimization, first of all, we focus on the distribution of the network in clusters taking into account, the most priority node as master of each cluster. The density of its neighborhood is the selection criterion. Second, the average speed of all the nodes, belonging to its cluster according to their speed of movement, represents the degree of mobility of the cluster, this value evaluated by the master of each cluster. In addition, the interception and control of the exchanged traffic of each cluster, this mission carried out by the cluster manager, in addition to the k-hop transmission. Among the essential information, different codes sent from the cluster master to its neighboring nodes in order to ensure security. 


\subsection{Clusters creation}

For reconstruct clusters, Figure 8 illustrates this operation, includes a cluster density and neighborhood in Figure 8(a) and the candidate cluster head nodes in Figure 8(b) and the cluster formation in Figure 8(c), the description of the algorithm detailing the headlines:

- $\quad$ Each node calculates the density of its neighborhood

- $\quad$ The node with the highest density, named as the cluster master

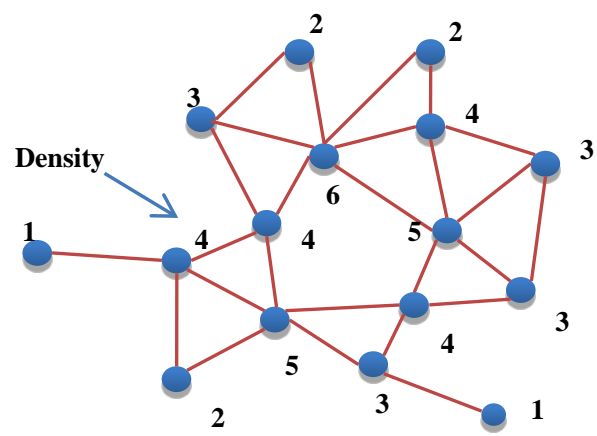

(a)

\begin{tabular}{|c|c|c|c|c|}
\hline ni & Nbr-set & D & CH & CH_D \\
\hline a & b1 & 1 & $\mathrm{~b}$ & 4 \\
\hline b & $a 1, c 2, e 4, d 5$ & 4 & $\mathrm{~d}$ & 5 \\
\hline $\mathbf{c}$ & b4, d5 & 2 & $\mathrm{~d}$ & 5 \\
\hline $\mathrm{d}$ & $\mathrm{b} 4, \mathrm{c} 2, \mathrm{e} 4,13, \mathrm{k} 4$ & 5 & $\mathrm{~d}$ & 5 \\
\hline e & $\mathrm{b} 2, \mathrm{~d} 5, \mathrm{f} 3, \mathrm{~h} 6$ & 4 & $\mathrm{~h}$ & 6 \\
\hline$f$ & $\mathrm{e} 4, \mathrm{~h} 6, \mathrm{~g} 2$ & 3 & $\mathrm{~h}$ & 6 \\
\hline g & $\mathrm{e} 4, \mathrm{f} 3, \mathrm{~h} 6$ & 2 & $\mathrm{~h}$ & 6 \\
\hline $\mathrm{h}$ & e4，f3， $\underset{i 5}{i 5}, \circ 2 ， p 4$ & 6 & $\mathrm{~h}$ & 5 \\
\hline$i$ & $\mathrm{~h} 6, \mathrm{k} 4, \mathrm{~m} 3, \mathrm{n} 3, \mathrm{p} 4$ & 5 & $\mathrm{~h}$ & 6 \\
\hline$j$ & 13 & 1 & 1 & 3 \\
\hline $\mathrm{k}$ & $\mathrm{d} 5,13, \mathrm{~m} 3, \mathrm{i} 5$ & 4 & $\mathrm{~d}$ & 5 \\
\hline 1 & $\mathrm{~d} 5, \mathbf{k} \mathbf{4}, \mathbf{j} 1$ & 3 & $\mathrm{~d}$ & 5 \\
\hline $\mathrm{m}$ & $i 5, \mathrm{k} 4, \mathrm{n} 3$ & 3 & $i$ & 5 \\
\hline $\mathrm{n}$ & $\mathrm{m3}, \mathrm{i5}, \mathrm{p} 4$ & 3 & $i$ & 5 \\
\hline$\circ$ & $\mathrm{p} 4, \mathrm{~h} 6$ & 2 & $\mathrm{~h}$ & 6 \\
\hline $\mathrm{p}$ & $\mathrm{h} 6, \mathrm{i} 5, \mathrm{n} 3, \mathrm{p} 4$ & 4 & $\mathrm{~h}$ & 6 \\
\hline
\end{tabular}

(b)

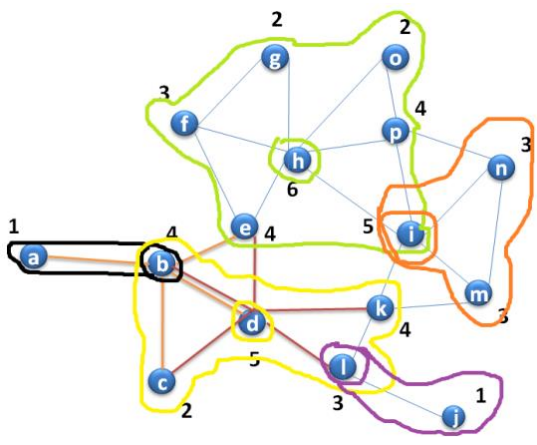

(c)

Figure 8. Cluster head selection and clusters formation; (a) cluster density and neighborhood, (b) candidate cluster head nodes and their density, (c) clusters construction and cluster head

\section{CONCLUSION}

In this work, we have developed a new model to refine the security and QoS MANET constraints by integrating the continuous network MSSP formulation of Hopfield $(\mathrm{CHN})$ in a big data environment with hadoop ecosystem. Integrated optimization is a result of the clustering technique, combining both the calculation of mobility as a basic metric and another metric ensuring the control of traffic passing through the cluster and measuring its volume in order to set up a multi-system -objective and polymorphic. The

Int J Artif Intell, Vol. 10, No. 2, June 2021: 458 - 466 
developed approach proposes two stages: the first acts at the level of the election of the OLSR master cluster in order to quickly complete the density using the CHN, by modeling the MSSP problem. Second, the objective is to optimize the precision making an efficient solution to the first neighborhood rank as a linear constraint, and at the end, find the resolution of the model using the CHN. We have proven that the MSSP model presented is essential to ensure the proper functioning of OLSR routing. in order to test the recommended results, computer treatments generated by the MSSP problem have been published. And as perspectives, in OLSR, the construction of clusters could be extended to reach the second rank of cluster nodes, with a metric representing the mobility speed of the cluster and add the parameter of the available bandwidth, enrich the OLSR protocol with new parameters namely multi-objective metrics. A research guideline consists in applying this approach to optimize the CHN coloring graphs, the best path following well defined criteria; moreover, this approach corrects the problems, in particular at the level of the Hadoop environment, improves the quadratic function.

\section{REFERENCES}

[1] H. Ehsan, F. A. Khan, "Implementation and Analysis of Routing Attacks in MANETs," 2012 IEEE 11th International Conference on Trust, Security and Privacy in Computing and Communications, 2012, pp. 1181-1187, doi: 10.1109/TrustCom.2012.199.

[2] A. Ez-zahout and J. Oubaha, "The Big Data-RTAP: Toward a Secured Video Surveillance System in Smart Environment," International Conference of Cloud Computing Technologies and Applications, vol. 49, 2019, pp. 142-149, doi: 10.1007/978-3-319-97719-5.

[3] B. Wu, J. Chen, J. Wu, and M. Cardei, "A Survey on Attacks and Countermeasures in Mobile Ad Hoc Networks," wireless/Mobile Network Security, pp. 103-135, 2007, doi: 10.1007/978-0-387-33112-6_5.

[4] N. Lakki, J. Oubaha, A. Ouacha, A. Habbani and J. Elabbadi, "The effect of the Multi-objective dynamic metric on the QoS and the energy in networks MANETs," International Journal of Recent Contributions from Engineering, Science \& IT (iJES), vol. 7, no. 2, pp. 30-48, 2019, doi: 10.3991/ijes.v7i2.10439.

[5] A. Barbir, S. Murphy, Y. Yang, "Generic Threats to Routing Protocols," IETF RFC4593. Status Informational, 2006, doi: $10.17487 / \mathrm{rfc} 4593$.

[6] I. Bekmezci, O. K. Sahingoz, S. Temel, "Flying ad-hoc networks (FANETs): A survey," Ad Hoc Networks, vol. 11, no. 3, pp. 1254-1270, 2013, doi: 10.1016/j.adhoc.2012.12.004.

[7] P. M. Talaván, J. Yàñez, "The generalized quadratic knapsack problem. A neuronal network approach," Neural Networks, vol. 19, no. 4, pp. 416-428, 2006, doi: 10.1016/j.neunet.2005.10.008.

[8] C. Rajarama, J. N. Sugatoor, T. Y. Swamy, "Random routing scheme with misleading dead ends," International Journal of Informatics and Communication Technology (IJ-ICT), vol. 8, no. 2, pp. 87-93, 2019, doi: 10.11591/ijece.v9i5.pp4176-4183.

[9] J. Jailton, T. Carvalho, J. Araújo, R. Francês, "Relay Positioning Strategy for Traffic Data Collection of Multiple Unmanned Aerial Vehicles Using Hybrid Optimization Systems: A FANET-Based Case Study", Wireless Communications and Mobile Computing, vol. 2017, 2017, Art. no. 2865482, doi: 10.1155/2017/2865482.

[10] B. Thiongane, A. Najih and G. Plateau, "An Adapted Step Size Algorithm for a 0-1 Biknapsack Lagrangean Dual," Annals of Operations Research, vol. 139, no. 1, pp. 353-373, 2005, doi: 10.1007/s10479-005-3454-x.

[11] S. Rosati, K. Krużelecki, G. Heitz, D. Floreano, B. Rimoldi, "Dynamic routing for flying ad hoc networks," IEEE Transactions on Vehicular Technology, vol. 65, no. 3, pp. 1690-1700, 2016, doi: 10.1109/TVT.2015.2414819.

[12] Y. Dehbi, N. Mikou, "Priority Assignment for Multimedia Packets Scheduling in MANET," 2008 IEEE International Conference on Signal Image Technology and Internet Based Systems, 2008, pp. 32-37, doi: 10.1109/SITIS.2008.81.

[13] A. V. Leonov, "Modeling of bio-inspired algorithms AntHocNet and BeeAdHoc for Flying Ad Hoc Networks (FANETs)," 2016 13th International Scientific-Technical Conference on Actual Problems of Electronics Instrument Engineering (APEIE), vol. 2, 2016, pp. 90-99, doi: 10.1109/APEIE.2016.7806419.

[14] L. Cui, F. R. Yu, and Q. Yan, "When big data Meets Software Defined Netwowork SDN for Big data and Big data for SDN," IEEE Network, vol. 30, no. 1, pp. 58-65, doi: 10.1109/MNET.2016.7389832.

[15] J. Oubaha, A. Echchaachoui, A. Ouacha, and M. Elkoutbi, "New Method: Mapping of 802.11e into MPLS Domains," International Conference on Digital Information Processing and Communications- ICDIPC 2011, vol. 189, 2011, pp. 470-483, doi: 10.1007/978-3-642-22410-2_41.

[16] J. Oubaha, A. Habbani and M. Elkoutbi, "New Approach: Mapping of 802.11e into MPLS Domains," The 2nd International Conference on Multimedia Computing and Systems (ICMCS'11), 2011, pp. 497-502, doi: 10.1109/ICMCS.2011.5945692.

[17] A. A. Cardinas, P. K. Mandhata, and S. Rajan, "Big data analytics for security intelligence," University of texas at Dallas@ cloud Security Alliance. pp: 1-22, 2013, doi: 10.12691/iteces-4-1-3.

[18] P. M. Talaván and J. Yànez, "A continuous Hpfield network equilibrium points algorithm," computers and operations research, vol. 32, no. 8, pp. 2179-2196, 2005, doi: 10.1016/j.cor.2004.02.008.

[19] Y. N. Prajapati, M. K. Srivastava, "Novel algorithms for protective digital privacy," IAES International Journal of Robotics and Automation, vol. 8, no. 3, pp. 184-188, 2019, doi: 10.11591/ijra.v8i3.pp184-188.

[20] A. A. Hussien, M. J. Marie, K. S. Gaeid, "Effect of fuzzy PID controller on feedback control systems based on wireless sensor network," International Journal of Electrical and Computer Engineering (IJECE), vol. 10, no. 3, pp. 2416-2424, 2020, doi: 10.11591/ijece.v10i3.pp2416-2425. 
[21] L. E. Aik, T. W. Hong, A. K. Junoh, "Distance weighted K-Means algorithm for center selection in training radial basis function networks," IAES International Journal of Artificial Intelligence (IJ-AI), vol. 8, no. 1, pp. 54-62, 2019, doi: 10.11591/ijai.v8.i1.pp54-62.

[22] A. Ullah, N. M. Nawi, J. Uddin, S. Baseer, A. H. Rashed, "Artificial bee colony algorithm used for load balancing in cloud computing: review," IAES International Journal of Artificial Intelligence (IJ-AI), vol. 9, no. 1, pp. 33-39, 2020, doi: 10.11591/ijai.v8.i2.pp156-167.

[23] S. Barhmi, O. El Fatni, "Hourly wind speed forecasting based on support vector machine and artificial neural networks," IAES International Journal of Artificial Intelligence (IJ-AI), vol. 8, no. 3, pp. 286-291, 2019, doi: 10.11591/ijai.v8.i3.pp286-291.

[24] A. Guha, "Prediction of bankruptcy using big data analytic based on fuzzy c-means algorithm," IAES International Journal of Artificial Intelligence (IJ-AI), vol. 8, no. 2, pp. 168-174, 2019, doi: 10.11591/ijai.v8.i2.pp168-174.

[25] N. M. Mahfuz, M. Yusoff, Z. Ahmad, "Review of single clustering methods," IAES International Journal of Artificial Intelligence (IJ-AI), vol. 8, no. 3, pp. 221-227, 2019, doi: 10.11591/ijai.v8.i3.pp221-227.

\section{BIOGRAPHIES OF AUTHORS}
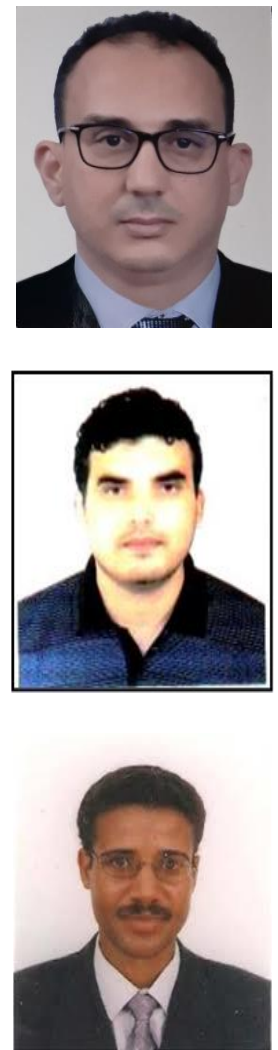

Jawad Oubaha is professor of computer science at the University of Mohammed-V Rabat (UM5) Morocco. He received his Ph. Doctorate degree from the ENSIAS School of Computer Engineering. Oubaha is a associated member of the Intelligent Processing and Security of Systems (IPSS) team of Computer Science Department at the FSR. His research is currently focused on optimizing the performance of routing protocols with machine learning in an Internet of Things (IoT) and Big Data environment. He is the author and co-author of several publications in international journals and conferences. Oubaha is a member of the organizing committees of several scientific events (Conferences and Congresses). He is also a member of the Technical Program Committee (TPC) of several international conferences and journals.

Noureddine Lakki got a specialist Master networks and telecommunications in 2009 in LABO STIC (Science Information Technology and Communication) faculty of sciences El jadida Morocco. He has thesis about mobility in ad hoc for the amelioration of performance of system MIS in laboratory LEC (Laboratory of Electronics and Telecommunication) in Graduate School of Engineering, EMI .He belongs to MIS (Mobile intelligent System) team. His research is currently focused on optimizing the performance of routing protocols in an Internet of Things (IoT), Mobile Edge Computing (MEC) and Mobile Ad-hoc Networks environment. He is also a member of the Technical Program Committee (TPC) of several international conferences and journals.

Ali Ouacha is professor of computer science at the Faculty of Science of Rabat (FSR) Universities Mohamed V (UM5) since 2018. He received his doctorate degree from the Mohammadia School of Engineering (EMI). Ouacha is a permanent member of the Intelligent Processing and Security of Systems (IPSS) team of Computer Science Department at the FSR. His research is currently focused on optimizing the performance of routing protocols in an Internet of Things (IoT), Mobile Edge Computing (MEC) and Mobile Ad-hoc Networks environment. He is the author and co-author of several publications in international journals and conferences. Ouacha is a member of the organizing committees of several scientific events (Conferences and Congresses). He is also a member of the Technical Program Committee (TPC) of several international conferences and journals. 